\title{
Descomposición de imágenes médicas 3D en sub-bandas a través de la transformada discreta de wavelet
}

\author{
Decomposition of 3D medical imaging in sub-band through \\ discrete wavelet transform
}

Pedro Freddy Huamaní Navarrete

\section{Resumen}

En los últimos años, los diagnósticos médicos a través de la interpretación de imágenes médicas digitales como las de gammagrafia, eco cardiografía, resonancia magnética y tomografia axial computarizada, se han acentuado cada vez más. Ello se debe, principalmente, a la diversidad de técnicas matemáticas de procesamiento digital de imágenes existentes; tal es el caso de la Transformada Discreta de Wavelet Directa (TDW) e inversa (TDWI), técnica que permite la disgregación en sub-bandas de imágenes 2D y 3D. De esta manera, en el presente artículo, se exhibe el procedimiento de obtención de la TDW sobre imágenes digitales en 3D, utilizando la Familia Wavelet Daubechies. Dicha TDW es aplicada sobre cada cuadro de la imagen médica $3 D$, logrando así una descomposición de la misma en varias sub bandas. Tanto el algoritmo de descomposición y de reconstrucción de las sub bandas, son propios y se encuentran programados en funciones que se ejecutan bajo la plataforma del Matlab. Asimismo, se muestra los resultados, tanto numéricos como visuales, sobre la imagen $3 D$ al momento de reconstruirla con la TDW inversa.

\section{Palabras claves}

Imágenes 3D, transformada discreta de wavelet, familia Daubechies.

\section{Abstract}

In recent years, medical diagnostics through the interpretation of digital medical images such as scintigraphy, echocardiography, magnetic resonance and computed tomography have increased even more. This is mainly due to the variety of mathematical techniques of existing digital image processing, such is the case of Discrete Wavelet Transform Direct (DWT) and inverse (TDWI), a technique that allows the breakdown into sub-band images $2 \mathrm{D}$ and $3 \mathrm{D}$. Thus, in this article, shows the procedure for obtaining the DWT on digital images in 3D, using Daubechies Wavelet Family. This DWT is applied to each frame of the $3 D$ medical imaging, thus a decomposition of it into several sub bands. Both the decomposition algorithm and reconstruction of the sub bands are unique and are programmed in functions that run under the platform of Matlab. It also shows the results, both numerical and visual, on the $3 D$ image when reconstructed with the inverse DWT.

1 Ingeniero electrónico. Magíster en Procesamiento de Señales y Control de Procesos. Docente URP 


\section{Key words}

$3 D$, discrete wavelet transform, Daubechies family.

\section{Introducción}

En la actualidad, el auge de la tecnología digital viene ayudando notablemente a los profesionales de la medicina. Es así, que a partir de la interpretación de imágenes médicas provenientes, por ejemplo, de una gammagrafía, eco cardiografía, resonancia magnética, tomografía axial computarizada, entre otras, es posible la realización de diversos diagnósticos; $y$, todo ello es viable, gracias a las variedades de técnicas matemáticas existentes para el procesamiento digital de imágenes, tal como es el caso de la Transformada Discreta de Wavelet (TDW) que ha resultado ser una técnica matemática utilizada para mejorar la calidad en una imagen, logrando mayor nitidez en las zonas de suma importancia para el diagnóstico médico; asimismo, con dicha transformada, se consigue eliminar o reducir relativamente el ruido proveniente de diversos medios; así como también, se logra reducir el número de bits por pixel, y con ello el beneficio en la transmisión o almacenamiento de imágenes médicas digitales en su formato propio denominado DICOM.

De esta manera, en este artículo, se muestra una introducción a la interpretación de imágenes médicas digitales bidimensionales (2D) y tridimensionales (3D) a través del software Matlab, tomándolos como matrices o arreglos de diferentes dimensiones. Posteriormente, se presenta el procedimiento de obtención de la TDW con fines de descomponer una imagen médica en uno o dos niveles con varias presentaciones de sub bandas.

Para ello, se presenta un código de programación desarrollado que se ejecutará en el software Matlab. Este código corresponde a los algoritmos de descomposición en sub-bandas, y respectiva reconstrucción de las mismas. Asimismo, se muestra la obtención de la relación señal a ruido pico (PSNR) con el objetivo de obtener una comparación numérica entre la imagen 3D original y la reconstruida, como complemento a la comparación visual que se realiza con ayuda de técnicas de renderización.

Es preciso señalar que en el presente artículo, solamente se aplica el algoritmo de cálculo de la TDW directa e inversa, basado en una programación propia y sin hacer uso de los comandos DWT2 e IDWT2 que posee el Wavelet Toolbox del software Matlab. De la misma manera, en ningún momento se realiza la codificación de los coeficientes de la TDW, por lo tanto se consigue una reconstrucción de la imagen prácticamente perfecta debido a las propiedades que poseen los filtros de descomposición y reconstrucción de cualquiera de las familias de wavelet existentes. 


\section{Aspectos Teóricos}

A continuación se definen algunos aspectos teóricos utilizados en el desarrollo de este artículo.

\section{- Estándar DICOM}

Es el acrónimo de Digital Imaging and Communication in Medicine (Imagen y Comunicación Digital en Medicina), y se define como un estándar reconocido a nivel mundial para el intercambio de imágenes médicas digitales. El estándar DICOM fue creado por el ACR (American College of Radiology) y NEMA (National Electrical Manufacturers Association) en el año 1993, y desde esa fecha viene utilizándose frecuentemente por muchos centros hospitalarios. Asimismo, se entiende como un estándar que surgió de las necesidades usuario-fabricante, y comunicación de equipos heterogéneos entre sí.

Los archivos DICOM se diferencian de los comúnmente JPG, GIF, TIFF, etc., desde el punto de vista de almacenamiento de información. Es decir, los archivos DICOM almacenan la data correspondiente a una imagen en particular, tal como lo hace JPG, GIF, etc. pero así mismo también almacenan datos particulares del paciente (nombre, edad, sexo, fecha de cumpleaños, etc.), nombre de la institución y alguna información adicional de suma importancia para el médico.

\section{- Imagen médica en 3D}

Una imagen médica se entiende como una representación espacial de la distribución de propiedades físicas en el interior del cuerpo humano, y es utilizada frecuentemente para el diagnóstico, monitoreo y planificaciones quirúrgicas. Entre los métodos más empleados para la captura de imágenes médicas, se tiene: la radiología, medicina nuclear o tomografía por emisión de positrones (PET), angiografía digital, tomografía axial computarizada (TAC) y la resonancia magnética (NMR). ${ }^{2}$

De esta manera, una imagen médica en 3D es producida a partir de un PET, TAC o NMR, y se obtiene al capturar imágenes bidimensionales de una sección de volumen del cuerpo humano. Es decir, a través de una secuencia de cortes paralelos y adyacentes, se da origen a una imagen en 3D que es posible visualizarla con la asistencia de algoritmos de reconstrucción; tal es el caso del algoritmo gráfico para computadoras, denominado Marching Cubes.

\section{- Imagen digital en Matlab}

Para el software Matlab, que es la herramienta computacional que se utiliza en este artículo, las imágenes digitales son interpretadas como matrices o arreglos. 
Para el caso de una imagen bidimensional a tonos de gris, la representación se da como una matriz de "M" filas y " $\mathrm{N}$ " columnas $(\mathrm{M} \times \mathrm{N})$. Por ejemplo, digitar en la ventana de comandos del Matlab lo siguiente:

$$
\begin{aligned}
& \text { >load imdemos } \\
& \text { > whos moon } \\
& \text { > } \mathrm{M}, \mathrm{N}]=\operatorname{size}(\text { moon }) ; \\
& \text { > } \text { figure(1) } \\
& \text { > >imshow(moon) }
\end{aligned}
$$

\section{\%ver la figura 01.}

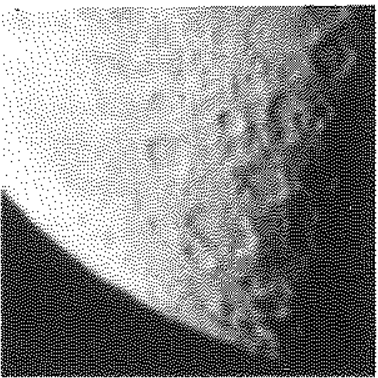

Figura 01. Representación de una imagen bidimensional en tonos de gris

Asimismo, para el caso de una imagen bidimensional a color, la representación se realiza como un arreglo conformado por tres matrices de " $\mathrm{M}$ " filas y " $\mathrm{N}$ " columnas cada una. Es decir, es un arreglo de $\mathrm{MxNx3}$. Las tres matrices juntas forman el color y hacen referencia al modelo RGB (rojo-verdeazul). Por ejemplo, digitar en la ventana de comandos del Matlab lo siguiente:

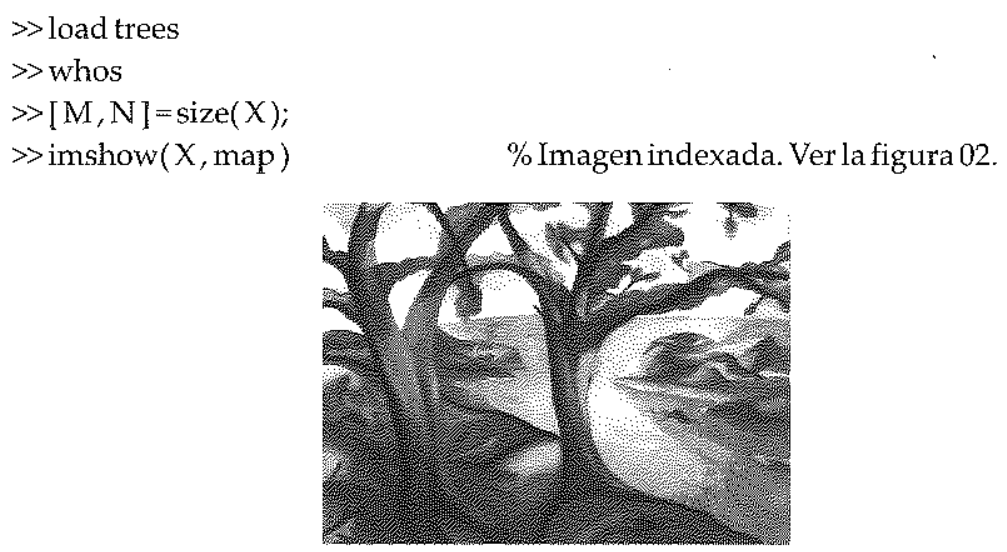

Figura 02. Representación de una imagen bidimensional a colores.

En este ejemplo del Matlab, la imagen a color está representada como imagen indexada. Es decir, conformada por dos matrices que señalarán el color (variable map) y su ubicación en la matriz principal (variable X). Por lo tanto, a continuación se emplea un comando o función para convertir una imagen indexada en una RGB. 


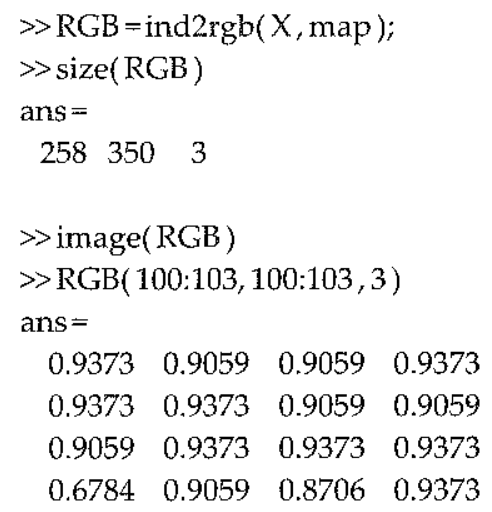

De igual manera, para el caso de una imagen tridimensional (3D) a tonos de gris, la representación se da como un conjunto de imágenes bidimensionales conformadas por " $\mathrm{M}$ " filas y " $\mathrm{N}$ " columnas cada una $(\mathrm{MxN} \times 1 \times \mathrm{F})$, donde $\mathrm{F}^{\prime \prime}$ representa el número de cuadros o frames. Por ejemplo, digitar en la ventana de comandos del Matlab lo siguiente:

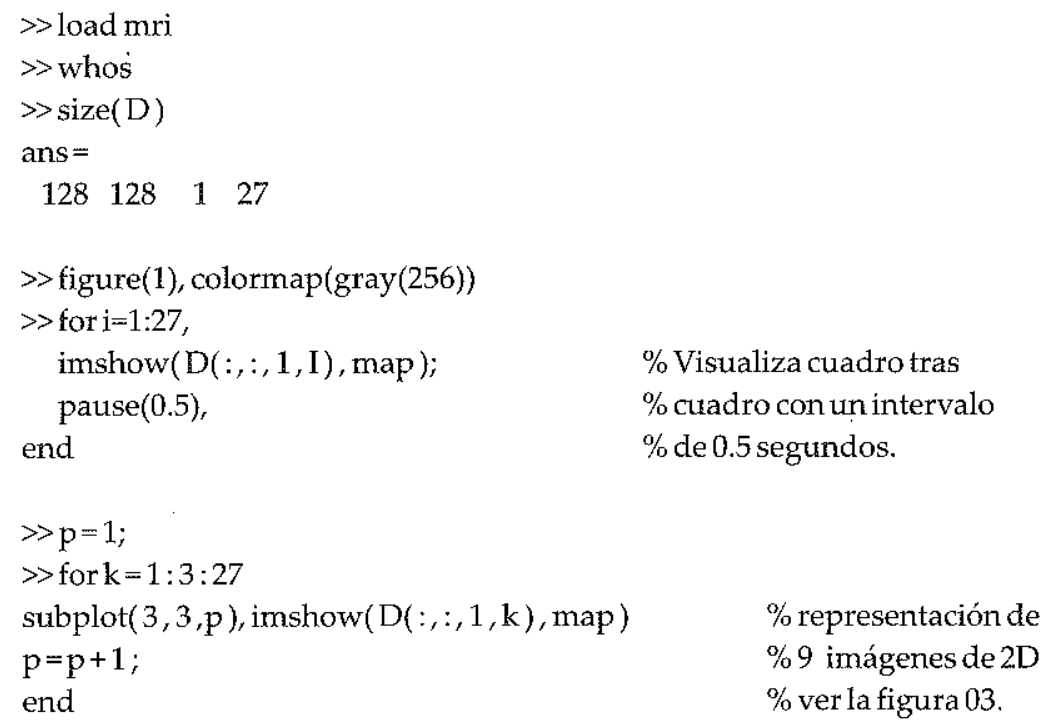

Para la visualización de la imagen médica en 3D, se procede a utilizar la función VOL3D, que hace uso de una técnica de mapeo para renderizar imágenes bidimensionales. La renderización se entiende como el proceso de generar una imagen tridimensional a partir de un modelo y utilizando una aplicación de computadora. 


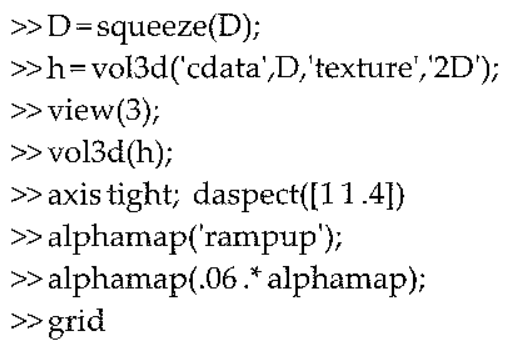

$\%$ ver la figura 04 ,

$\%$ representación en $3 \mathrm{D}$.
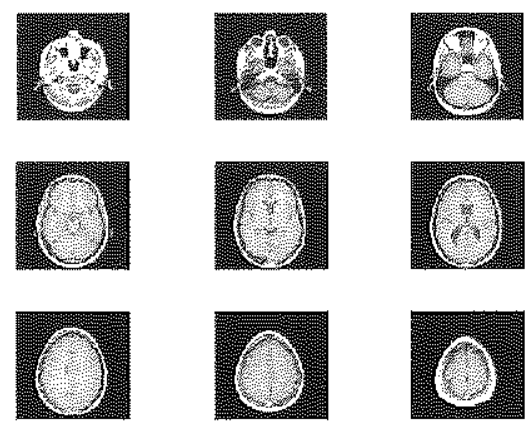

Figura 03. Visualización de nueve imágenes médicas 2D correspondientes a la variable $\mathrm{D}$ del ejemplo anterior.

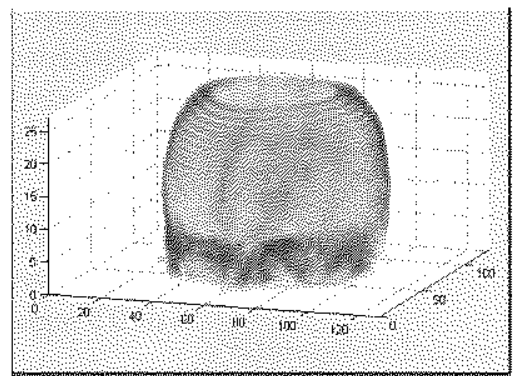

Figura 04. Visualización de imagen médica en 3D utilizando las 27 imágenes bidimensionales.

\section{- Transformada Wavelet}

La Transformada Wavelet de una función perteneciente al espacio de las funciones cuadráticamente integrables, $L^{2}\{R\}$, es su descomposición en una base formada por expansiones, contracciones y traslaciones de una única función madre $(\mathrm{t})$, llamada como Wavelet. ${ }^{5}$

Asimismo, se puede mostrar, que para señales o imágenes digitales, la Transformada Wavelet es un caso especial de bancos de filtros críticamente decimados. De esta manera, en la figura 05, se muestra la relación existente con los bancos de filtros para el caso de imágenes digitales en 2D, 


\section{Implementación del Modelo}

La implementación de este modelo de descomposición en sub-bandas, fue realizado sobre la imagen ejemplo que cuenta el software Matlab. Para ello, se procedió a realizar la descomposición en uno y dos niveles sobre cada uno de los 27 cuadros por el cual está conformada esta imagen médica 3D. Dentro de todas las familias Wavelets existentes, se eligió la familia Daubechies con un banco de filtros de orden 12, con la finalidad de contar con el tamaño apropiado del filtro cuando se realice la operación de filtrado en cada imagen. Es decir, como cada cuadro tiene un tamaño de 128x128 pixeles, al aplicar la descomposición en un nivel, se obtendrán imágenes de $1 / 4$ del tamaño de la imagen original (64×64). Mientras que para el caso de la descomposición en dos niveles, las imágenes resultantes tendrán un tamaño igual a 1/16 del tamaño de la imagen original (32×32). Igualmente, los filtros de descomposición y reconstrucción de la familia Daubechies, poseen la propiedad de ortogonalidad. Esto indica que su producto interno es igual a cero.

\begin{tabular}{|c|c|}
\hline $\begin{array}{l}\gg[\text { pbd, pad, pbr, par }]=\text { wfilters('db12'); } \\
\gg\left[\mathrm{pbd}^{*} \text { pad' }^{\prime}, \mathrm{pbr}^{*} \mathrm{par}^{\dagger}\right] \\
\text { ans }=\end{array}$ & $\begin{array}{l}\% \text { banco de filtros de la familia } \\
\% \text { Daubechies del Toolbox } \\
\% \text { Wavelet del Matlab. }\end{array}$ \\
\hline$>[\mathrm{PBD}, \mathrm{F}]=$ freqz $(\mathrm{pbd}, 1)$ & \\
\hline$\gg[P A D, F]=\operatorname{freqz}(\operatorname{pad}, 1)$ & $\begin{array}{l}\text { \% Transformada Discreta de } \\
\% \text { Fourier (TDF) a los filtros }\end{array}$ \\
\hline$\gg[P B R, F]=\operatorname{freqz}(\mathrm{pbr}, 1)$ & \% de descomposicióny \\
\hline$\gg[P A R, F]=$ freq $z($ par, 1$)$ & \% reconstrucción. \\
\hline $\begin{array}{l}>P \mathrm{PBD}=20^{*} \log 10(\mathrm{abs}(\mathrm{PBD})) ; \\
>>\mathrm{PAD}=20^{*} \log 10(\mathrm{abs}(\mathrm{PAD})) ; \\
>>\mathrm{PBR}=20^{*} \log 10(\mathrm{abs}(\mathrm{PBR})) ; \\
>>\mathrm{PAR}=20^{*} \log 10(\mathrm{abs}(\mathrm{PAR})) ;\end{array}$ & $\begin{array}{l}\text { \% obteniendo el } \\
\% \text { módulo de la TDF. }\end{array}$ \\
\hline$\gg$ subplot $(2,2,1), \operatorname{plot}(F, P B D)$, grid & \% en estas líneas se aprecia \\
\hline$\gg$ subplot $(2,2,2), \operatorname{plot}(F, P A D)$, grid & $\%$ la respuesta en frecuencia \\
\hline$\gg$ subplot $(2,2,3), \operatorname{plot}(F, P B R)$, grid & $\%$ del banco de filtros de la \\
\hline$\gg$ subplot $(2,2,4), \operatorname{plot}(F$, PAR $)$, grid & $\%$ familia Daubechies. \\
\hline
\end{tabular}

Con los filtros de descomposición y reconstrucción definidos, se procede a descomponer cada cuadro de la imagen 3D, en uno y dos niveles. Para esto, se utiliza la función desarrollada en este trabajo que lleva por nombre DESCOMPONE. Tener en cuenta que se debe ingresar el número de niveles de descomposición. Asimismo, antes de descomponer la imagen, se crea un arreglo de ceros para ìr colocando en él cada una de las descomposiciones por cada nivel.

De esta manera, si se desea apreciar la descomposición de cada cuadro en forma aislada, bastará con visualizarlo del arreglo principal TDW_1_N o TDW_2_N, según el número de niveles de descomposición. 
A continuación se muestra el código empleado en el Matlab, para la obtención de la descomposición en sub-bandas, o aplicación de la Transformada Discreta de Wavelet Directa.

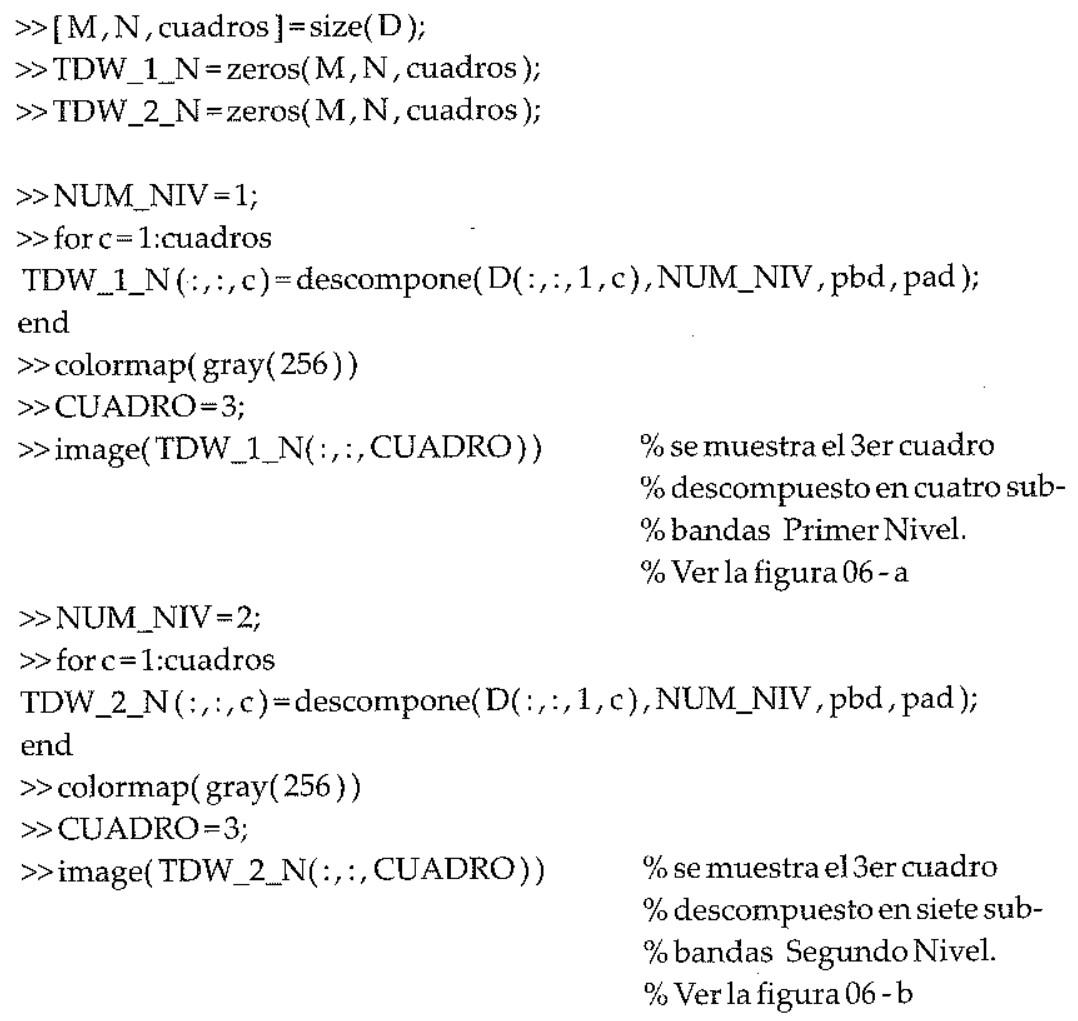

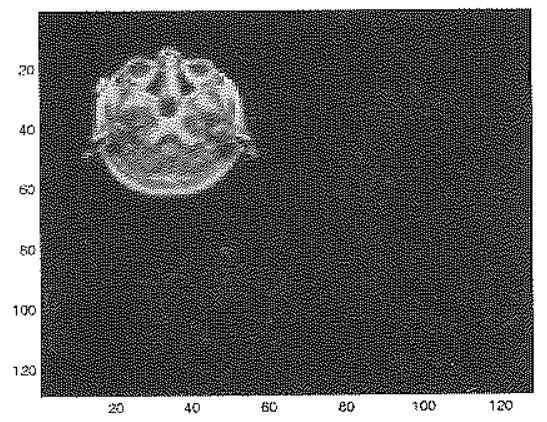

(a)

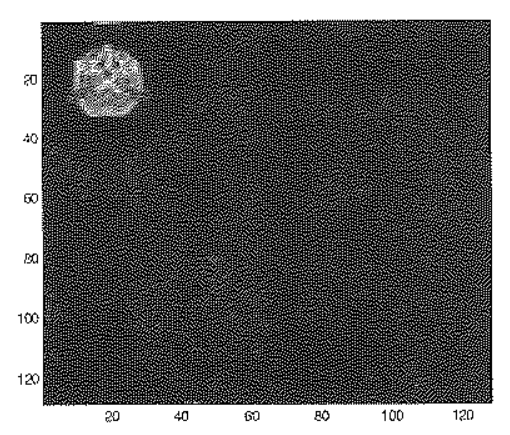

(b)

Figura 06. Descomposición. a) Primer nivel con 4 sub-bandas. b) Segundo nivel con 7 sub-bandas. 
Obtenida la descomposición, se procede a realizar la etapa inversa que corresponde a la aplicación de la Transformada Discreta de Wavelet Inversa, o también denominada reconstrucción.

Para la realización de la reconstrucción, también se procede a crear dos arreglos que contengan las 27 imágenes reconstruidas. A continuación, se muestra el código empleado en el Matlab para lograr esta segunda etapa. Asimismo, se presenta el uso de la función desarrollada en este trabajo, el cuallleva por nombre RECONSTRUYE.

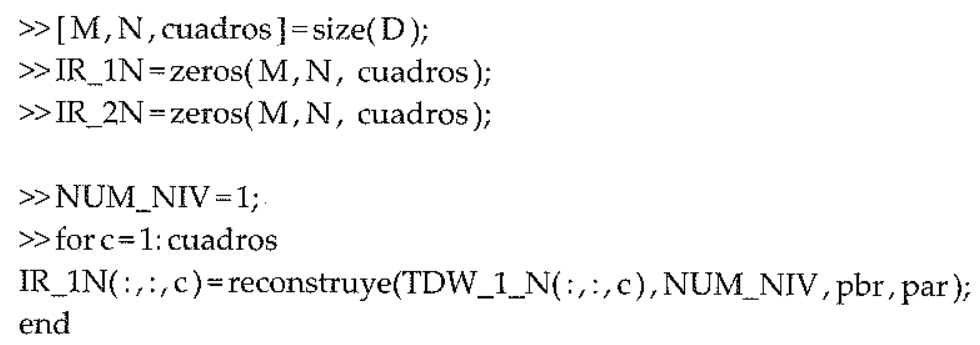

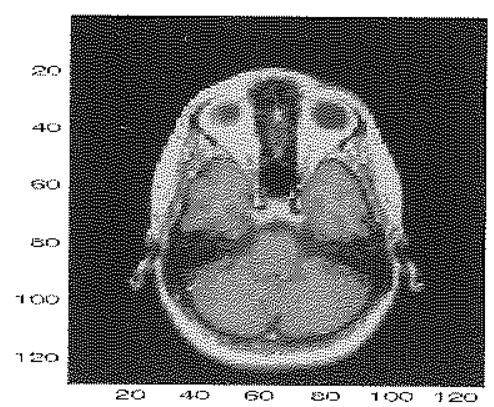

(a)

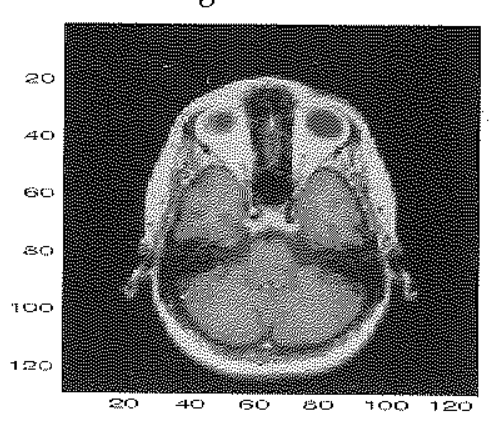

(b)

Figura 07. Reconstrucción. a) Desde el primer nivel de descomposición. b) Desde el segundo nivel de descomposición. 


\section{Resultados}

Para conocer el grado de distorsión creado al momento de aplicar la TDW en uno y dos niveles, se utiliza el parámetro de medición muy utilizado en trabajos de investigación relacionados a la compresión de imágenes digitales, la relación señal a ruido pico que se define en la ecuación $01{ }^{7}$.

$$
P S N R=10 \times \log _{1 \times 1}\left[\begin{array}{c}
\left(2^{N^{*} B F P}-1\right)^{2} \\
\frac{1}{M \times N} \sum_{i=1}^{M-1} \sum_{j=1}^{N-1}\left(I o_{i, j}-I r_{i, j}\right)^{2}
\end{array}\right]
$$

Donde:

$\begin{array}{lll}\operatorname{Io}(i, j) & : & \text { representa a la imagen original } \\ \operatorname{Ir}(i, j) & : & \text { representa a la imagen reconstruida } \\ \mathrm{MyN} & : & \text { cantidad de filas y columnas } \\ \mathrm{N} \text {-BPP }: & \text { número de bits por pixel. }\end{array}$

Esta relación de señal a ruido pico se utiliza comúnmente en imágenes bidimensionales, pero como en este trabajo se realiza la descomposición seguido de la reconstrucción sobre 27 imágenes bidimensionales, la relación PSNR se obtiene para cada una de las imágenes o cuadros por la cual está compuesta la imagen $3 \mathrm{D}$.

Es necesario aclarar que en este artículo solo se presenta la aplícación de la TDW directa e inversa, sin llegar a realizar la codificación de los coeficientes obtenidos. Por lo tanto, la relación PSNR obtenida es alta debido a que no existe pérdida alguna cuando se aplica la TDW directa; asimismo, la TDW inversa reconstruye completamente cada uno de los cuadros por el cual se encuentra conformada la imagen en 3D. A continuación, en la figura 08, se muestra un gráfico de la relación PSNR obtenida por cada cuadro, cuando se realiza la descomposición en 07 sub-bandas diferentes.

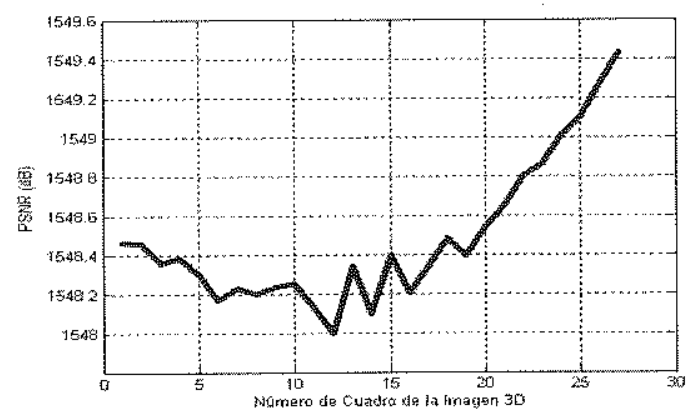

Figura 08. Gráfico de la relación PSNR para la descomposición y reconstrucción en dos niveles de cada cuadro de la imagen 3D.

Es preciso aclarar que para el caso de la descomposición en un solo nivel, la TDW inversa proporcionará cuadros prácticamente iguales, en donde su relación PSRN tiene un valor bastante elevado. Por tal motivo no se procede a representar su gráfica. 
Por otro lado, para el caso de una descomposición-reconstrucción en dos niveles, la relación PSNR promedio será igual a $1548 \mathrm{~dB}$.

Realizada la reconstrucción o TDW inversa a cada una de los 27 cuadros que contiene la imagen 3D, se procede a presentar la imagen en 3D después de aplicar un algoritmo de renderizado, tal como se realizó en la sección anterior, Imagen Digital en Matlab. Seguidamente, la figura 09 representa dicho resultado mostrando las imágenes $3 \mathrm{D}$ reconstruidas después de la descomposición en uno y dos niveles, respectivamente.

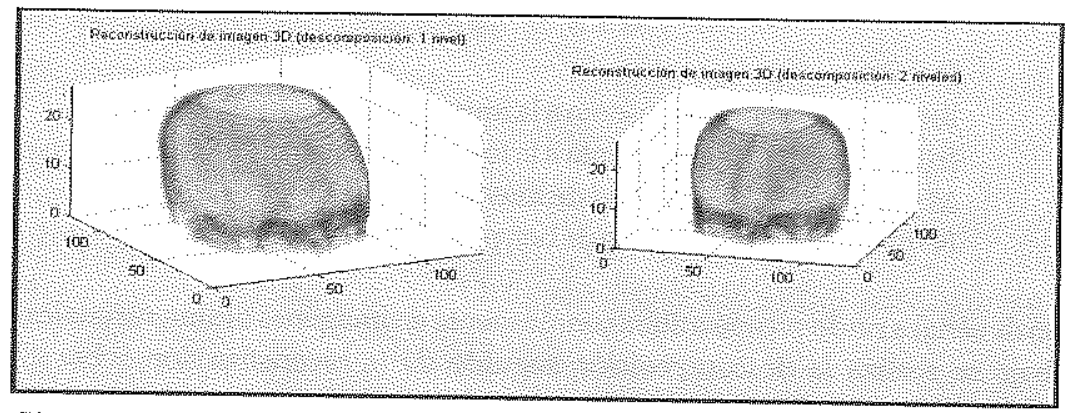

Figura 09. Reconstrucción. a) Desde el primer nivel de descomposición. b) Desde el segundo nivel de descomposición.

\section{Discusión}

Son muchas las familias de wavelets que existen en la actualidad, así tenemos por ejemplo: la familia Biorthogonal, Coiflets, Symlets, entre otras; donde la diferencia se encuentra en las características particulares del banco de filtros utilizado, tanto para la descomposición, como para la reconstrucción. Asimismo, dentro de cada familia, es posible hallar filtros de diversos números de retardos o de orden. Estos filtros de descomposición y reconstrucción, tienen la característica de poseer una respuesta finita al impulso, lo que asegura su estabilidad dentro del plano $Z$. De esta manera, es viable la aplicación de la TDW directa e inversa utilizando las otras familias Wavelets con diferente número de retardos, lo cual da origen a nuevos resultados que podrían mejorar o empeorar el procesamiento sobre la imagen digital que se viene tratando.

\section{CONCLUSIONES}

Entre las conclusiones más resaltantes de este trabajo, citamos las siguientes:

> La relación señal a ruido pico es relativamente alta, debido a que las imágenes fueron descompuestas y luego reconstruidas sin haber sido codificadas. Esto demuestra la efectividad del banco de filtros de reconstrucción perfecta.

- El aumento del número de niveles de descomposición origina mayor error en la imagen reconstruida, pues el error se propaga desde el último nivel hacia el primero.

$>$ Las imágenes digitales en 3D se caracterizan por poseer abundante información, pues se 
encuentran representadas por múltiples imágenes 2D. Por lo tanto, el procesamiento sobre este grupo de imágenes demandará mayor tiempo computacional del requerido comúnmente sobre imágenes bidimensionales.

\section{REFERENCIAS BIBLIOGRÁFICAS}

Ramirez Diniz, Barros da Silva e Lima NeTTo. 2004. "Processamento Digital de Sinais. Projeto e Análise de Sistemas". Editorial Bookman. Brasil.

VETTERLI, M. \& HERLEY, C. 1992. Wavelets and filters banks: Theory and design. IEEE Transactions on Signal Processing, 40, 2207-2232.

WAVELET TOOLBOX. Versión 4.1.Agosto 2007. The MathWorks Inc.

NAtional Electrical MANUfacturers Association. 2004. "Digital Imaging and Communications in Medicine (DICOM)". Rosslyn, Virginia 22209. USA.

ARCHITECTURE RE-USE COMMUNICATIONS. 1997. "DICOM Cook Book for Implementations in Modalities". Philips Medical Systems.

GARRIDO, D. P., "Laplacian Pyramid Coding Using Scalar and Vector Quantization," Rio de Janeiro, ICASSP-90 Technical Program Committee.

OPPENHEIM, AlAN V. \& SCHAFER, RONALD W. 1989. "Discrete-time Signal Processing". Editorial Prentice Hall.

http:/www.mathworks.com/matlabcentral/fileexchange/22940-vol3d-v2/content/vol3d.m By Oliver Wood ford.

http://www.ate.uniovi.es/14005/documentos/clases\%20pdf/imagenes\%20medicas.pdf

http:/www.alegsa.com.ar/Dic/renderizacion.php 